\title{
KELVIN PRINCIPLE FOR A CLASS OF SINGULAR EQUATIONS
}

\author{
ABDULLAH ALTIN
}

Faculty of Science

University of Ankara

Besevler, Ankara, Turkey

EUTIQUIO C. YOUNG

Department of Mathematics

Florida State University

Tallahassee, Florida 32306

(Received September 7, 1988)

ABSTRACT. The classical Kelvin principle concerns invariance of solutions of the Laplace equation with respect to inversion in a sphere. By employing a hyperbolicpolar coordinate system, the principle is extended to cover a class of singular equations, which include the ultrahyperbolic equation.

KEY WORDS AND PHRASES. Kelvin principle, Laplace equation, ultrahyperbolic equation, Lorentzian distance.

1980 AMS SUBJECT CLASSIFICATION CODES. 35L10, 35C99.

1. INTRODUCTION.

As is well known the classical Kelvin principle introduced in 1847 (Thomson [1]) concerns solutions of the Laplace equation. For solutions of some class of elliptic differential equations and their iterated forms in $n$ independent variables, $n \geq 2$, the extension of Kelvin principle is usually proved using rectangular coordinates (Diaz and Martin [2], Germain and Bader [3], Huber [4], Weinstein [5] ). In 1960

a generalization of Kelvin principle was established by Weinstein [5] for the equation

$$
\sum_{i=1}^{n}\left(\frac{\partial^{2} u}{\partial x_{i}^{2}}+\frac{k_{i}}{x_{i}} \frac{\partial u}{\partial x_{i}}\right)=0,-\infty<k_{i}<\infty
$$

using polar coordinates.

Following Weinstein method we shall give in this paper a new formulation of Kelvin principle for solutions of the class of singular partial differential equations

$$
L(u)=\sum_{i=1}^{n}\left(\frac{\partial^{2} u}{\partial x_{i}^{2}}+\frac{\alpha_{i}}{x_{i}} \frac{\partial u}{\partial x_{i}}\right)-\sum_{j=1}^{m}\left(\frac{\partial^{2} u}{\partial y_{j}^{2}}+\frac{\beta_{j}}{y_{j}} \frac{\partial u}{\partial y_{j}}\right)+\frac{1}{r^{2}} P(u)=0
$$

where $\alpha_{i}(l \leq i \leq n)$ and $\beta_{j}(l \leq j \leq m)$ are real parameters, $r$ is the lorentzidn metric defined by

$$
r^{2}=\sum_{i=1}^{n} x_{i}^{2}-\sum_{j=1}^{m} y_{j}^{2}
$$


and $\mathrm{P}$ is a general linear operator of arbitrary order $\mathrm{q}$ in the variables $\mathrm{z}_{1}, \mathrm{z}_{2}$, $\ldots, z_{p}$ vanishing for $u=0$.

The domain of the operator $\mathrm{L}$ is the set of all real valued functions $u(x, y, z)$ of class $c^{2}(D) \cap c^{q}(\Omega)$, where $x=\left(x_{1}, \ldots, x_{n}\right), y=\left(y_{1}, \ldots, y_{m}\right)$ and $z=\left(z_{1}, \ldots, z_{p}\right)$ denote points in $R^{n}, R^{m}$ and $R^{P}$, respectively, and $D \times \Omega$ is a regularity domain of $\mathrm{u}$ in $\mathrm{R}^{\mathrm{n}+\mathrm{m}} \times \mathrm{R}^{\mathrm{P}}$.

2. HYPERBOLIC-POLAR COORDINATE SYSTEM FOR EQUATION (1.1).

First let us consider the $n+m$-dimensional Laplace operator

$$
\Delta u=\sum_{i=1}^{n} \frac{\partial^{2} u}{\partial x_{i}^{2}}+\sum_{j=1}^{m} \frac{\partial^{2} u}{\partial x_{n+j}^{2}}
$$

and introduce the polar coordinates

$$
\begin{aligned}
& x_{1}=r \cos \theta_{1} \cos \theta_{2} \ldots \cos \theta_{m-1} \cos \theta_{m} \cos \theta_{m+1} \cdots \cos \theta_{m+n-2} \cos \theta_{m+n-1} \\
& x_{2}=r \cos \theta_{1} \cos \theta_{2} \ldots \cos \theta_{m-1} \cos \theta_{m} \cos \theta_{m+1} \cdots \cos \theta_{m+n-2} \sin \theta_{m+n-1} \\
& x_{3}=r \cos \theta_{1} \cos \theta_{2} \ldots \cos \theta_{m-1} \cos \theta_{m} \cos \theta_{m+1} \cdots \sin \theta_{m+n-2} \\
& x_{n}=r \cos \theta_{1} \cos \theta_{2} \ldots \cos \theta_{m-1} \cos \theta_{m} \sin \theta_{m+1} \\
& x_{n+1}=r \cos \theta_{1} \cos \theta_{2} \ldots \cos \theta_{m-1} \sin \theta_{m} \\
& x_{n+m-1}=r \cos \theta_{1} \sin \theta_{2} \\
& x_{n+m}=r \sin \theta_{1}
\end{aligned}
$$

where $0 \leq \theta_{j} \leq \pi$ for $j=1, \ldots, n+m-2,0 \leq \theta_{n+m-1} \leq 2 \pi$ and

$$
r=\left(x_{1}^{2}+\ldots+x_{n+m}^{2}\right)^{\frac{1}{2}}
$$

Under this change of variables, the polar form of the Laplace operator is given by

$$
\Delta u=\sum_{i=1}^{n+m} \frac{\partial^{2} u}{\partial x_{i}^{2}}=\frac{\partial^{2} u}{\partial r^{2}}+\frac{n+m-1}{r} \frac{\partial u}{\partial r}+\frac{1}{r^{2}} \Phi_{1}(u)
$$

where the operator $\Phi_{1}$ depends only on the variables $\theta_{1}, \cdots, \theta_{n+m-1}$ and $r$ is the Euclidean distance given by $(2.3)$.

Now in (2.1) and (2.2) let $x_{n+j}=i y_{j}$ and $\theta_{j}=i_{j}$ for $j=i, \ldots, m$ with $i=V_{-1}$ and let $\theta_{m+j}=\psi_{j}$ for $j=1, \ldots, n-1$. Since $\left(\partial / \partial x_{n+j}\right)^{2} u=-\left(\partial / \partial y_{j}\right)^{2} u$, $\cos \left(i \phi_{j}\right)=\operatorname{ch} \phi_{j}$ and $\sin \left(i \phi_{j}\right)=i \operatorname{sh} \phi_{j}$, the operator (2.1) reduces to the ultrahyperbolic operator

$$
\square u=\sum_{i=1}^{n} \frac{\partial^{2} u}{\partial x_{i}^{2}}-\sum_{j=1}^{m} \frac{\partial^{2} u}{\partial y_{j}^{2}}
$$

On the other hand, the polar coordinate system (2.2) takes the form 


$$
\begin{aligned}
& x_{1}=r \operatorname{ch} \phi_{1} \operatorname{ch} \phi_{2} \ldots \operatorname{ch} \phi_{m-1} \operatorname{ch} \phi_{m} \cos \psi_{1} \ldots \cos \psi_{n-2} \cos \psi_{n-1} \\
& x_{2}=r \operatorname{ch} \phi_{1} \operatorname{ch} \phi_{2} \ldots \operatorname{ch} \phi_{m-1} \operatorname{ch} \phi_{m} \cos \psi_{1} \ldots \cos \psi_{n-2} \sin \psi_{n-1} \\
& x_{3}=r \operatorname{ch} \phi_{1} \operatorname{ch} \phi_{2} \ldots \operatorname{ch} \phi_{m-1} \operatorname{ch} \phi_{m} \cos \psi_{1} \ldots \sin \psi_{n-2} \\
& x_{n}=r \operatorname{ch} \phi_{1} \operatorname{ch} \phi_{2} \ldots \operatorname{ch} \phi_{m-1} \operatorname{ch} \phi_{m} \sin \psi_{1} \\
& y_{1}=r \operatorname{ch} \phi_{1} \operatorname{ch} \phi_{2} \ldots \operatorname{ch} \phi_{m-1} \operatorname{sh} \phi_{m} \\
& y_{2}=r \operatorname{ch} \phi_{1} \operatorname{ch} \phi_{2} \ldots \operatorname{sh} \phi_{m-1} \\
& y_{m-1}=r \operatorname{ch} \phi_{1} \operatorname{sh} \phi_{2} \\
& y_{m}=r \operatorname{sh} \phi_{1}
\end{aligned}
$$

where $r$ is the Lorentzian distance given by (1.2). We refer to this as "polarhyperbolic transformation". In the polar-hyperbolic coordinate system, the operator (2.5) assumes the form

$$
\square u=\sum_{i=1}^{n} \frac{\partial^{2} u}{\partial x_{i}^{2}}-\sum_{j=1}^{m} \frac{\partial^{2} u}{\partial y_{j}^{2}}=\frac{\partial^{2} u}{\partial r}+\frac{n+m-1}{r} \frac{\partial u}{\partial r}+\frac{1}{r^{2}} \Phi_{2}(u)
$$

where $\Phi_{2}$ depends only on the variables $\phi_{1}, \ldots, \phi_{m}, \psi_{1}, \ldots, \psi_{n-1}$ and $r$ is given in $(1.2)$.

For example, the polar forms of $\Delta u$ for $n=2,3$ are given by

$$
\begin{aligned}
& \Delta \mathrm{u}=\mathrm{u}_{\mathrm{rr}}+\frac{1}{\mathrm{r}} \mathrm{u}_{\mathrm{r}}+\frac{1}{\mathrm{r}^{2}} \mathrm{u}_{\theta \theta} \\
& \Delta \mathrm{u}=\mathrm{u}_{\mathrm{rr}}+\frac{2}{\mathrm{r}} \mathrm{u}_{\mathrm{r}}+\frac{1}{\mathrm{r}^{2}}\left(\mathrm{u}_{\theta \theta}+\frac{\cos \theta}{\sin \theta} u_{\theta}+\frac{1}{\sin ^{2} \theta} u_{\phi \phi}\right)
\end{aligned}
$$

The corresponding forms for the hyperbolic equations are given by

$$
\begin{aligned}
& \square u=u_{r r}+\frac{1}{r} u_{r}-\frac{1}{r^{2}} u \\
& \square u=u_{r r}+\frac{2}{r} u_{r}-\frac{1}{r^{2}}\left(u_{\theta \theta}+\frac{\operatorname{sh} \theta}{\operatorname{ch} \theta} u_{\theta}-\frac{1}{\operatorname{ch}^{2} \theta} u_{\phi \phi}\right)
\end{aligned}
$$

where $\square u=u_{x x}+u_{y y}-u_{z z}, r^{2}=x^{2}+y^{2}-z^{2}$ and $x=r \operatorname{ch} \theta \cos \phi, y=r \operatorname{ch} \theta \sin \phi$, $z=r \operatorname{sh} \theta$.

3. A FUNDAMENTAL THEOREM AND MAIN RESULT.

In [5], using his main three recursion formulas, Weinstein gave the followir, theorem which will be used to establish our main result.

THEOREM 1. Let $v=v\left(r, \psi_{1}, \ldots, \psi_{n-1}\right)$ satisfy the differential equation

$$
\frac{\partial^{2} v}{\partial r^{2}}+\frac{k}{r} \frac{\partial v}{\partial r}=\frac{1}{r^{2}} \Phi(v)
$$

where $k$ is a real or complex number and $\Phi$ is a linear differential operator vanishing for $v=0$ which is independent of the variable $r$. Then $\rho^{1-k} w$ satisfies the same 
equation (3.1) in the variables $\rho, \psi_{1}, \ldots, \psi_{n-1}$, where $\rho=1 / r$ and $\mathrm{w}\left(\rho, \psi_{1}, \ldots, \psi_{\mathrm{n}-1}\right)=\mathrm{v}\left(1 / \rho, \psi_{1}, \ldots, \psi_{\mathrm{n}-1}\right)$

Using Theorem 1 we can now establish an extension of Kelvin principle to ultrahyperbolic equations :

THEOREM 2. Let $\mathrm{u}=\mathrm{u}\left(\mathrm{x}_{1}, \ldots, \mathrm{x}_{\mathrm{n}}, \mathrm{y}_{1}, \ldots, \mathrm{y}_{\mathrm{m}}, \mathrm{z}_{1}, \ldots, \mathrm{z}_{\mathrm{p}}\right)$ be a solution of the equation (1.1). Then

$$
w=r^{-\lambda} u\left(\frac{x_{1}}{r^{2}}, \ldots, \frac{x_{n}}{r^{2}}, \frac{y_{1}}{r^{2}}, \ldots, \frac{y_{m}}{r^{2}}, z_{1}, \ldots, z_{p}\right)
$$

is also a solution of the same equation (1.1), where

$$
\lambda=n+m-2+\sum_{i=1}^{n} \alpha_{i}+\sum_{j=1}^{m} B_{j}
$$

and $\mathrm{r}$ is the Lorentzian distance defined by (1.2).

PROOF. Let us consider the polar-hyperbolic transformation (2.6) which can be written in the following form

$$
\begin{aligned}
x_{i} & =r f_{i}(\phi, \psi), \quad i=1, \ldots, n \\
y_{j} & =r g_{j}(\phi, \psi), \quad j=1, \ldots, m
\end{aligned}
$$

where the notations $f_{i}(\phi, \psi), g_{j}(\phi, \psi)$ or without subscripts $f(\phi, \psi), g(\phi, \psi)$ denote functions of $\phi_{1}, \ldots, \phi_{m}, \psi_{1}, \ldots, \psi_{n-1}$.

We note that from (3.4) we have

$$
\begin{aligned}
& \frac{\partial x_{k}}{\partial x_{j}}=\frac{\partial x_{k}}{\partial r} \frac{\partial r}{\partial x_{j}}+\sum_{i=1}^{m} \frac{\partial x_{k}}{\partial \phi_{i}} \frac{\partial \phi_{i}}{\partial x_{j}}+\sum_{i=1}^{n-1} \frac{\partial x_{k}}{\partial \psi_{i}} \frac{\partial \psi_{i}}{\partial x_{j}}=\delta_{j k} \\
& \frac{\partial y_{k}}{\partial y_{j}}=\frac{\partial y_{k}}{\partial r} \frac{\partial r}{\partial y_{j}}+\sum_{i=1}^{m} \frac{\partial y_{k}}{\partial \phi_{i}} \frac{\partial \phi_{i}}{\partial y_{j}}=\delta j k
\end{aligned}
$$

where $\delta_{j k}$ is the Kronecker delta. From (1.2) we have

$$
\frac{\partial r}{\partial x_{j}}=\frac{\partial x_{j}}{\partial r}(1 \leq j \leq n) \text { and } \frac{\partial r}{\partial y_{j}}=\frac{-y_{j}}{r}(1 \leq j \leq m)
$$

and we may express the partial derivatives $\partial \phi_{i} / \partial x_{j}, \partial \psi_{i} / \partial x_{j}$ and $\partial \phi_{i} / \partial y_{j}$ as a quotient the denominator of which is the Jacobian of the transformation (2.6). It can be shown that

$$
\frac{\partial\left(x_{1}, \ldots, x_{n}, y_{1}, \ldots, y_{m}\right)}{\partial\left(r, \phi_{1}, \ldots, \phi_{m}, \psi_{1}, \ldots, \psi_{n-1}\right)}=r^{n+m-1} h(\phi, \psi)
$$

The numerator of this quotient contains obviously only a factor $r^{n+m-2}$, hence 


$$
\frac{\partial \phi_{i}}{\partial x_{j}}=\frac{1}{r} F_{j i}(\phi, \psi), \frac{\partial \psi_{i}}{\partial x_{j}}=\frac{1}{r} G_{j i}(\phi, \psi), \frac{\partial \phi_{i}}{\partial y_{j}}=\frac{1}{r} H_{j i}(\phi, \psi)
$$

On the other hand, since

$$
\begin{aligned}
& \left.\frac{\partial u}{\partial x_{j}}=\frac{x_{j}}{r} \frac{\partial u}{\partial r}+\frac{1}{r} \sum_{i=1}^{m} \frac{\partial u}{\partial \phi_{i}} F_{j i}(\phi, \psi)+\sum_{i=1}^{n-1} \frac{\partial u}{\partial \psi_{i}} G_{j i}(\phi, \psi)\right) \\
& \frac{\partial u}{\partial y_{j}}=-\frac{y_{j}}{r} \frac{\partial u}{\partial r}+\frac{1}{r} \sum_{i=1}^{m} \frac{\partial u}{\partial \phi_{i}} H_{j i}(\phi, \psi)
\end{aligned}
$$

we see that

$$
\begin{aligned}
& \frac{1}{x_{j}} \frac{\partial u}{\partial x_{j}}=\frac{1}{r} \frac{\partial u}{\partial r}+\frac{1}{r^{2}} \Psi_{1}(u), \quad 1 \leq j \leq n \\
& \frac{1}{y_{j}} \frac{\partial u}{\partial y_{j}}=-\frac{1}{r} \frac{\partial u}{\partial r}+\frac{1}{r^{2}} \Psi_{2}(u), \quad 1 \leq j \leq m
\end{aligned}
$$

where the operators $\Psi_{1}$ and $\Psi_{2}$ depend only on the variables $\phi_{1}, \ldots, \phi_{m}, \psi_{1}, \ldots, \psi_{n-1}$. If we substitute the expressions (2.7) and (3.5) into (1.1), then our equation (1.1) becomes

$$
\frac{\partial^{2} u}{\partial r}+\frac{1}{r}\left(n+m-1+\sum_{i=1}^{n} \alpha_{i}+\sum_{j=1}^{m} \beta_{j}\right) \frac{\partial u}{\partial r}+\frac{1}{r^{2}} \Psi(u)=0
$$

Since $1-\left(n+m-1+\sum_{i=1}^{n} \alpha_{i}+\sum_{j=1}^{m} \beta_{j}\right)=-\lambda$, by Theorem 1

$$
w=r^{-\lambda} u\left(\frac{x_{1}}{r^{2}}, \ldots, \frac{x_{n}}{r^{2}}, \frac{y_{1}}{r^{2}}, \ldots, \frac{y_{m}}{r^{2}}, z_{1}, \ldots, z_{p}\right)
$$

satisfies the equation (1.1). Here we note that, since

$$
\rho^{2}=\sum_{i=1}^{n}\left(x_{i} / r^{2}\right)^{2}-\sum_{j=1}^{m}\left(y_{j} / r^{2}\right)^{2}=\frac{1}{r^{2}}
$$

the substitution $\rho=1 / r$ in the solution $u$ means replacing the variables $x_{i}$ and $y_{j}$ by $x_{i} / r^{2}$ and $y_{j} / r^{2}$, respectively.

4. REMARKS.

(i) We note that, since the $r$ defined by (1.2) is not real for $\Sigma_{i=1}^{n} x_{i}^{2}<$ $\sum_{j=1}^{m} y_{j}^{2}$, the solutions of $(1.1)$ is valid only in the domain $D x \Omega$, where

$$
D=D_{n} \times D_{m}=\left\{(x, y): x \in D_{n}, y \in D_{m}, \sum_{i=1}^{n} x_{i}^{2}>\sum_{j=1}^{m} y_{j}^{2}\right\}
$$

is a hyperconoidal domain in $R^{n+m}$. Here $D_{n}$ and $D_{m}$ are the spherical domains centered at the origins of $R^{n}$ and $R^{m}$, respectively, and $\Omega^{m} \subset R^{p}$ is the regularity domain of $\mathrm{u}$ with respect to the variable $z$. 
(ii) In equation (1.1) if we have addition instead of subtraction of the two summations, then Theorem 2 remains valid, where

$$
r^{2}=\sum_{i=1}^{n} x_{i}^{2}+\sum_{j=1}^{m} y_{j}^{2}
$$

This includes Weinstein's [5] and Altin's [2] results.

(iii) In the special case $\mathrm{Pu}=\gamma \mathrm{u}$ where $\gamma=$ const. the formula (3.2) gives the result obtained in [2].

(iv) If we multiply both sides of the equation (1.1) by -1 , we get the equation

$$
-L(u)=\sum_{j=1}^{m}\left(\frac{\partial^{2} u}{\partial y_{j}^{2}}+\frac{\beta_{j}}{y_{j}} \frac{\partial u}{\partial y_{j}}\right)-\sum_{j=1}^{n}\left(\frac{\partial^{2} u}{\partial x_{j}^{2}}+\frac{\alpha_{j}}{x_{j}} \frac{\partial u}{\partial x_{j}}\right)+\frac{1}{r^{2}} P(u)=0
$$

where $r_{1}^{2}=\Sigma_{j=1}^{m} y_{j}^{2}-\Sigma_{j=1}^{n} x_{j}^{2}=-r^{2}$. This shows that if $u\left(x_{1}, \ldots, x_{n}, y_{1}, \ldots, y_{m}, z_{1}, \ldots, z_{p}\right)$ is a solution of the equation (1.1), then by Theorem 2

$$
w_{1}=r_{1}^{-\lambda} u\left(\frac{x_{1}}{r^{2}}, \ldots, \frac{x_{n}}{r^{2}}, \frac{y_{1}}{r^{2}}, \ldots, \frac{y_{m}}{r^{2}}, z_{1}, \ldots, z_{p}\right)
$$

is also a solution of the same equation (1.1), where $\lambda$ is given by (3.3). It is clear that this solution is valid in a different domain where $r_{1}^{2}>0$, that is, $r^{2}<0$.

(v) It is clear that by a simple linear transformation, Theorem 2 also holds for a more general equation of the form

$$
\sum_{i=1}^{n} a_{i}^{2}\left(\frac{\partial^{2} u}{\partial \zeta_{i}^{2}}+\frac{\alpha_{i}}{\zeta_{i}-\zeta_{i}^{0}} \frac{\partial u}{\partial \zeta_{i}}\right)-\sum_{j=1}^{m} b_{j}^{2}\left(\frac{\partial^{2} u}{\partial n_{j}^{2}}+\frac{\beta_{j}}{n_{j}-n_{j}^{0}} \frac{\partial u}{\partial n_{j}}\right)+\frac{1}{r^{2}} P(u)=0
$$

where $a_{i}, b_{i}, a_{i}, \beta_{i}$ are real parameters $\left(a_{j} \neq 0, b_{i} \neq 0\right), \zeta^{0}=\left(\zeta_{1}^{0}, \ldots, \zeta_{n}^{0}\right)$ and $n^{\circ}=\left(n_{1}^{\circ}, \ldots, n_{m}^{\circ}\right)$ are fixed points in $D_{n}$ and $D_{m}$, respectively. Here

where

$$
w=r^{-\lambda} u\left(\frac{\zeta_{1}-\zeta_{1}^{\circ}}{a_{1} r^{2}}, \ldots, \frac{\zeta_{n}-\zeta_{n}^{\circ}}{a_{n} r^{2}}, \frac{n_{1}-n_{1}^{\circ}}{b_{1} r^{2}}, \ldots, \frac{n_{m}-n_{m}^{\circ}}{b_{m} r^{2}}, z_{1}, \ldots, z_{p}\right)
$$

$$
r^{2}=\sum_{i=1}^{n}\left(\frac{\zeta_{i}-\zeta_{i}^{\circ}}{a_{i}}\right)^{2}-\sum_{j=1}^{m}\left(\frac{n_{j}^{-n_{j}^{\circ}}}{b_{j}}\right)^{2}
$$

\section{REFERENCES}

1. THOMSON, W. Extraits de deus Lettres Addressees a M. Liouville, J. Math. Pures Appl. 12(1847), 256.

2. ALTIN, A. Some Expansion Formulas for a Class of Singular Partial Differential Equations, Proc. Amer. Math. Soc. 85(1982), 42-46.

3. GERMAIN, P. and BADER, R. Sur le Problems de Tricomi, Rend. Circ. Mat. Palermo $\underline{2}(1953)$, 53-70.

4. HUBER, A. Some Results on Generalized Axially Symmetric Potential Theory, Proc. Conf. Partial Diff. Eqs., University of Maryland, 1956, 147-155.

5. WEINSTEIN, A. On a Singular Differential Operator, Ann. Mat. Pura Appl. 49(1960), $359-365$.

6. DIAZ, J. B. and MARTIN, M. H. Riemann's Method and the Problem of Cauchy, II. The Wave Equation in $n$ Dimension, Proc. Amer. Math. Soc. 3(1952), 476-483. 


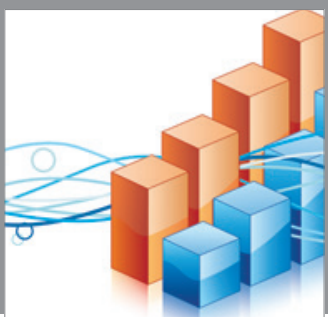

Advances in

Operations Research

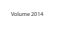

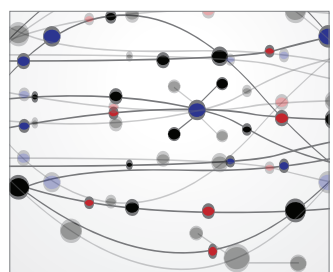

\section{The Scientific} World Journal
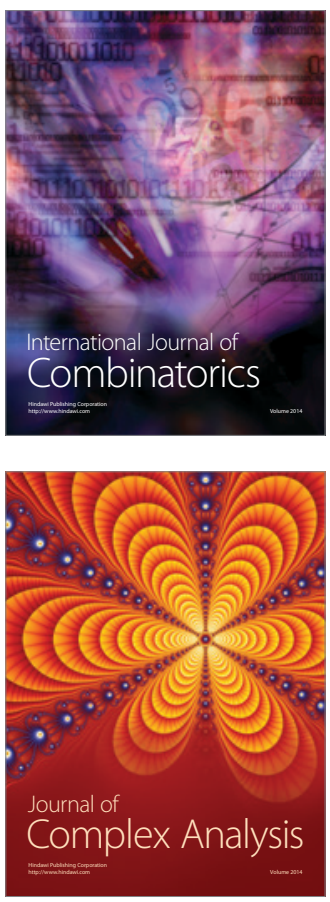

International Journal of

Mathematics and

Mathematical

Sciences
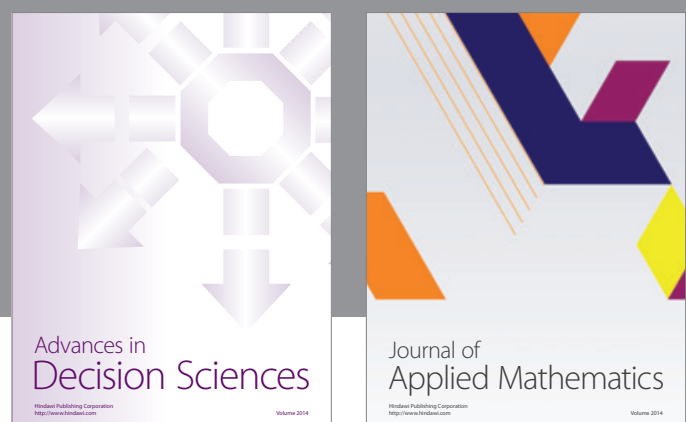

Journal of

Applied Mathematics
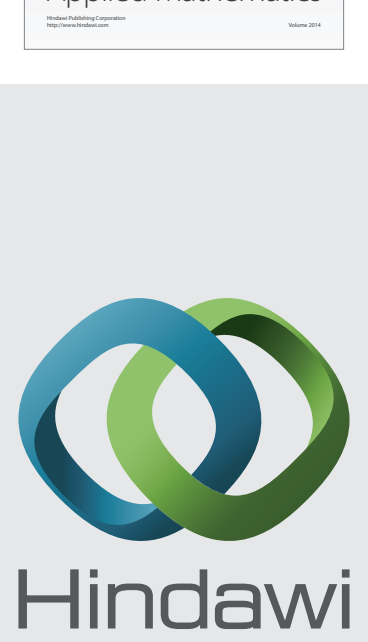

Submit your manuscripts at http://www.hindawi.com
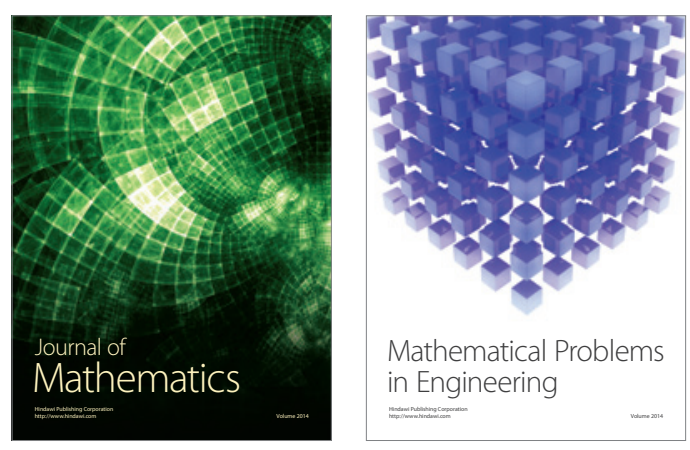

Mathematical Problems in Engineering
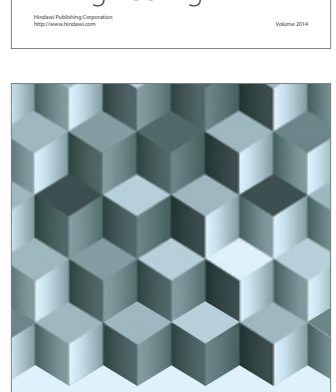

Journal of

Function Spaces
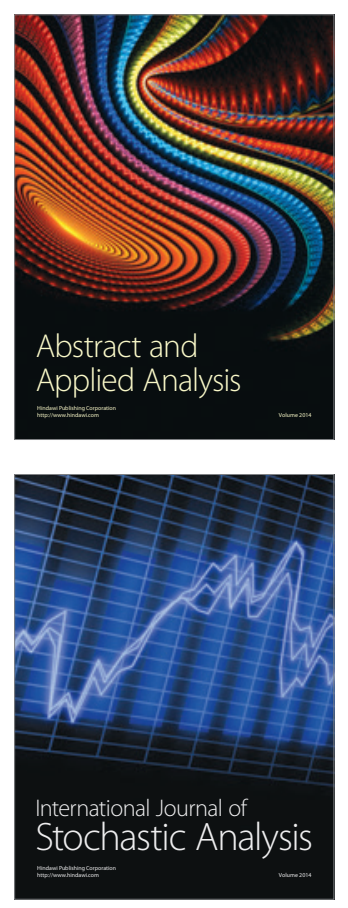

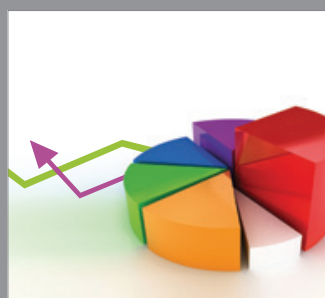

ournal of

Probability and Statistics

Promensencen
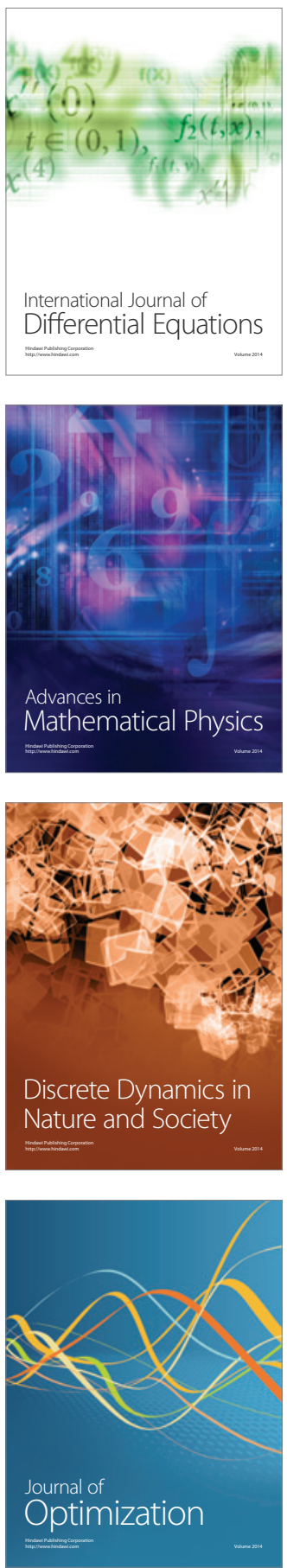